\title{
Occurrence Date Range
}

National Cancer Institute

\section{Source}

National Cancer Institute. Occurrence Date Range. NCI Thesaurus. Code C93608.

The date and time span in which the event began and ended. 